\title{
A Flow Model for the Settling Velocities of non Spherical Particles in Creeping Motion, Part II
}

\author{
Y. Mendez \\ Independent Research Group, Ottawa, Ontario, K2B 5W9, Canada \\ Corresponding Author Email: info@independentresearchgroup.ca
}

(Received March 3, 2010; accepted October 9, 2011)

\begin{abstract}
This paper follows previous work regarding the settling velocity of non spherical particles in creeping motion. In the previous work it was found that the shear stress in the fluid is opposed the mass of the fluid. The challenge of the shear stress by the mass imply a pressure gradient by default, i.e. the transfer of the shear stress to the mass is in the form of a surface stress $(\mathrm{Pa} / \mathrm{m})$, perpendicular to the shear stress, controlled by the mechanics of viscosity. The dynamics are triggered by the wall shear of the particle. Examination using measured settling velocities shows that the pressure gradient is a unique value for the fluid properties, so that the computed shear stress equal to the viscosity when the velocity gradient is equal to unit and the velocity is satisfied simultaneously, hence, defining the size of the expansion about the shear stress. We learned that application of the viscosity principle demand simultaneous consideration of the volumetric nature of the pressure gradient and the geometry dependence of the velocity gradient. We here undertake an examination to find how the pressure gradient is controlled by the fluid properties and a solution is reached. The solution is in good agreement with published experimental data. In addition we pursued further improvement of the relationships derived previously with further simplification.
\end{abstract}

Keywords: Sedimentation, Settling, Wall shear, Expansion, non spherical particles, Aspect ratio.

\section{NOMENCLATURE}

$\begin{array}{ll}A o & \text { surface area of a an oblate spheroid } \\ A S S & \text { specific surface area } \\ b & 1 / 2 \text { the thickness of an oblate spheroid } \\ a_{r} & \text { aspect ratio } \\ b & \text { radius of an oblate spheroid } \\ e & \text { tributary ratio } \\ e_{I} & \text { pseudo tributary ratio } \\ e_{I \max } & \text { maximum pseudo tributary ratio } \\ \mathrm{e}_{\max } & \text { maximum tributary ratio } \\ G s & \text { specific gravity of solid } \\ G_{f} & \text { specific gravity of fluid } \\ f(a, b) & \text { function of a and b } \\ g & \text { acceleration due to gravity }\end{array}$

\section{INTRODUCTION}

The wide variety of fields of science and engineering that may benefit from the study of the settling of particles in creeping motion have been widely pointed out in almost all research papers and books in low Reynolds hydrodynamics. Happel et al. (1983) lists chemical, civil and mining engineering among other fields.

In previous work Mendez, Y. (2011) on the settling velocity of isolated spherical and non spherical

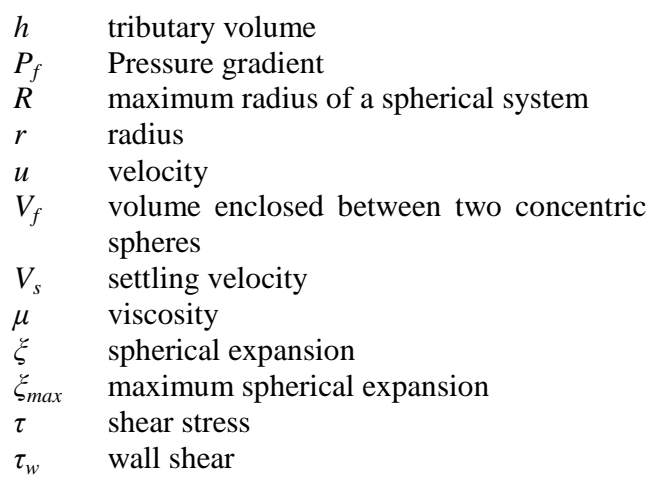

particles moving within a quiescent fluid continuum in creeping motion it was concluded that, given a force at the wall of the settling object, the fluid properties are entirely responsible for controlling the dynamics and the required dimensions of the ambient fluid so that a force, equal to the driving force is mobilized at the wall. Although simple, a deep examination of the dynamics is required to undertake on further insights of the dynamics of the ambient expansion about settling spheres later examined and evaluated in this paper. The general 
intent is to derive a rational approach to address practical problems in sedimentation. This goal moves within the rational guidelines quoted by $\mathrm{H}$, Henning Winter (2011), "Proposed research should contribute to basic understanding; thus enabling the better design; predictability; efficiency; and control of systems that involve fluids".

In the previous work examination of the quantities involved led to the conclusion that the driving force is shear stress. It was envisioned that there is mass quantity associated with any square meter of solid in $\mathrm{kg} / \mathrm{m}^{2}$ as provided by the inverse of the quantity known as the specific surface area ASS. Further examination leads to the conclusion that this quantity is a tributary mass of solids on the square meter $M_{T S}$ in $\mathrm{kg} / \mathrm{m}^{2}$ and the effect of gravity follows as $M_{T S} g$. Is then a trivial matter to demonstrate that for solids of specific gravity $G_{S}$ within a fluid of specific gravity $G_{F}$, the gravitational effect on the submerged tributary mass of the particle is $\left(\left(G_{S^{-}}\right.\right.$ $\left.\left.G_{F}\right) / G_{S}\right) M_{T S} g . \quad$ In the creeping regime this quantity just needs to be small enough so that the shear stresses in the fluid (comparable in magnitude) control the motion through the skin by wall shear $\tau_{W}$. One can verify that the settling velocity for spherical particles $V s$ is proportional to $\tau_{w}{ }^{2}$ in the form $V s \approx 0.0775 \tau_{w}{ }^{2}$. The next step is to note the relationship between the ambient fluid and the wall shear under the dynamics of viscous flows. In this context, there is no deliberate attempt to introduce a pressure gradient; however, we have studied how in viscous flows shear stresses are induced by a pressure gradient. One should hence wonder, what the response of the fluid is, when the shear stress is the driving force. The introduction of the pressure gradient in the relationships is rather confusing because it is often associated with external forces but a close examination of the concept itself provides clarification. The pressure gradient is a surface force acting in the direction of the pressure loss in $\mathrm{Pa} / \mathrm{m}$ and its definition implies that the net force acting on a volume of fluid (under the effect of the pressure gradient) equal the pressure gradient times the volume of the fluid in identical computation that a body force computes the force when multiplied by the volume or the mass under its influence. Although the definition is important in its understanding, one can give, from a practical stand point, the same consideration to the pressure gradient and a body force in $\mathrm{N} / \mathrm{m}^{3}$. To avoid any confusion, it will be referred to in $\mathrm{Pa} / \mathrm{m}$, however, readers not strictly in the field of fluid dynamics may wish to refer to it as a body force for comfort. On the question raised, it was concluded that the relationship between the wall shear and the ambient fluid prescribed by the dynamics of the viscosity principle dictate the mobilization of a surface force or pressure gradient $P_{f}$ who's plane of action is perpendicular to the direction of the velocity and the shear stress. From conventional mechanics it is easy to conclude that the shear stress in the fluid is being opposed by its own mass and that the transfer of the shear stress to the mass is in the form of a surface stress $(\mathrm{Pa} / \mathrm{m})$, perpendicular to the shear stress, controlled by the mechanics of the viscosity principle. The magnitude of this quantity is unknown; however, one can find its value, with measured settling velocities $V s$ from the following relationship:

$V_{s}=\frac{-3 \tau_{w} r_{s}}{2 \mu}\left(\frac{1}{e_{\max }}+\frac{2}{3}-\frac{\left(1+e_{\max }\right)^{\frac{2}{3}}}{e_{\max }}\right)$

where:

$r_{s}=$ the radius of the solid sphere,

$e_{\max }=$ the maximum tributary ratio, and

$\mu=$ the viscosity,

which is the equation for the settling velocity of spheres derived from the previous dynamics and appropriate geometric considerations. Solving Eq. 1 for $e_{\max }$, we find $e_{\max }=16.34$ with all other known quantities and compute the pressure gradient as the ratio of the shear stress by its tributary volume. $P_{f}=3 \tau_{W} /\left(e_{\max } r_{s}\right)=991 P a / m$ for fluid properties, density and viscosity at $15 \mathrm{C}^{\mathrm{o}}(\mu=$ $0.001139 \mathrm{~Pa}-s$ and $\rho_{f}=999.3 \mathrm{~kg} / \mathrm{m}^{3}$ ). The reader should refer to derivation of Eq. 29 in the previous work for the definitions of $\tau_{W}$ and $e_{\max }$ for spheres. As a result, the settling velocity at a given temperature and fluid can be computed as:

$V_{s}=\frac{-P_{f} r_{s}^{2}}{2 \mu}\left(1+\frac{2 e_{\max }}{3}-\left(1+e_{\max }\right)^{\frac{2}{3}}\right)$

Equation 2 solves for the velocity at the wall of the settling sphere but the entire velocity profile can be computed from:

$u=\frac{-P_{f}}{2 \mu}\left(\frac{r^{2}}{3}+\frac{2 R^{3}}{3 r}-R^{2}\right)$

where:

$u=$ velocity

$r=$ the radius of any spherical surface within the ambient fluid,

$R=$ the radius of the spherical system containing the ambient fluid and the solid sphere, where the velocity is cero.

The relationships between brackets in Eq. 3, in further discussion, the spherical expansion $\xi$, contains the geometric considerations to compute the velocity associated with any shear stress within the spherical ambient fluid. The relationship between brackets in Eq. 2, in further discussion, the maximum spherical expansion $\xi_{\max }$, allows for the computation of the velocity at the wall.

The dependence of the pressure gradient, on a plane normal to the shear stress, in the fluid properties, namely, viscosity and density; and how it influence the expansion so that the fluid mobilize the driving force at the wall of a settling particle is the subject of this paper.

Equation 2 is the result of the sound application of basic concepts in fluid mechanics, namely, the pressure gradient and viscosity. Its soundness is 
verified on conventional mechanics by the preservation of the integrity and significance of their computational advantages. In this context, there will be no further examination of the basic mechanics. If not already apparent, the reader is encouraged to conduct an examination.

\section{THE EFFECT OF THE DRIVING FORCE}

Consider the definition of the tributary ratio as the ratio of the volume of the ambient fluid to the volume of the solid sphere:

$e_{\max }=\frac{\frac{4}{3} \pi R^{3}-\frac{4}{3} \pi r_{s}^{3}}{\frac{4}{3} \pi r_{s}^{3}}=\frac{R^{3}-r_{s}^{3}}{r_{s}^{3}}$

Based on the tributary ratio simple definitions for the distance between the solid sphere to the boundary of the spherical ambient fluid $h_{\text {gmax }}=$ $r_{s}\left(\left(1+e_{\max }\right)^{1 / 3}-1\right)$ and the tributary volume of fluid $h_{\max }=\left(e_{\max } r_{s}\right) / 3$ overlying any square meter of particle, both inherently being considered in the derivation of Eq. 1, can be obtained. The maximum expansion groups the geometric considerations required to compute the shear stress and the velocity at a point across the ambient fluid coinciding with the surface of the solid sphere. The effect of the driving force in Eq. 2 is in the definition of the magnitude of $e_{\max }$. Here we note in general form the relationship between the wall shear and the pressure gradient as:

$\frac{\tau_{w}}{h_{\max }}=P_{f}$

which we further develop for understanding as:

$$
P_{f}=\frac{M_{T S} \frac{\left(G_{s}-G_{f}\right)}{G_{s}} g}{e_{\max } \frac{M_{T S}}{\rho_{s}}}=\frac{\left(G_{s}-G_{f}\right)}{e_{\max } G_{s}} g \rho_{s}
$$

For spheres the same conclusion arises as:

$$
P_{f}=\frac{\frac{r_{s} \rho_{s}}{3} \frac{\left(G_{s}-G_{f}\right)}{G_{s}} g}{\frac{e_{\max } r_{s}}{3}}=\frac{\left(G_{s}-G_{f}\right)}{e_{\max } G_{s}} g \rho_{s}
$$

where:

$\rho_{s}=$ the density of the solid sphere,

$g=$ the acceleration due to gravity

We conclude, from Eqs. 6 and 7 that the tributary ratio is just so much greater as the driving force, which are both, in proportion to the radius for spheres and Eq. 2 computes the velocity for all particle sizes. The resisting force is the pressure gradient and the dynamics of the transfer of the force to the sphere is viscosity.

From Eq. 1 we found $e_{\max }=16.34$ for particles of sand with $G s=2.65$ and the pressure gradient can also be computed from Eq. 7 at $991 \mathrm{~Pa} / \mathrm{m}$. Note that the effect of the specific gravity, or relative density, as provided by Eq. 6, is an increase or decrease in the tributary ratio, and hence the expansion.

With regard to the break down of the creeping regime, it appears to occur when the velocity gradient reaches about $140 \mathrm{~s}^{-1}$, which is slightly above the velocity gradient mobilized by a sand particle of radius $r_{s}=25 \mu \mathrm{m}$ and specific gravity equal to 2.65 in water at $20 C^{o}$ computed as $\tau_{W} / \mu=134$.

In this section, it can be seen that the experimental data confirm "the preservation of the integrity and significance of the computational advantages" of the concepts of pressure gradient and viscosity in the flow model studied on this paper and published in previous work. The conclusions derived will be considered factual in the following sections.

\section{The Pressure Gradient}

Let us consider the ambient fluid about a settling sphere and the dynamics envisioned in our flow model. Let us ignore the solid surface and consider a shear surface midway within the ambient fluid. The value of the shear stress on the given surface depends on the shear rate and the viscosity. Above and below the shear surface the pressure gradient becomes entirely defined by the fluid properties. Returning to the fluid layer bounded to the solid surface, which is in no way different than the layer midway within the ambient pressure gradient we note that the driving force just needs to be small enough to trigger the dynamics and does not play a role on its value. Although, under the basic concepts of fluid mechanics and the experimental evidence, the previous insights appear rather conclusive, none of the developments, along the derivation of Eq. 2, provide apparent indication on how the pressure gradient is defined by the fluid properties.

In this section we undertake an examination of the mass, volume, density, velocity gradient and viscosity quantities involved in the dynamics within the ambient fluid in order to achieve an understanding on how the pressure gradient is controlled by the fluid properties. The findings are later validated with published data. Except, otherwise specified, when computations are involved water properties at $15 C^{\circ}$ are considered, namely, $\mu=0.001139 \mathrm{~Pa}-\mathrm{s}$ and $\rho_{f}=999.3 \mathrm{~kg} / \mathrm{m}^{3}$.

Let us write in general form the equation for the settling velocity of an isolated particle moving through a quiescent fluid continuum:

$\mu \frac{d u}{d r}=P_{f} h$

where:

$h=$ the tributary volume

Eq. 8 can also, for clarity, be presented as:

$\frac{\mu}{P_{f}}=\frac{h}{d u / d r}$ 
The ratio $\mu / P_{c}$ is unique to the fluid at a given temperature. There is a corresponding tributary volume for every value of velocity gradient. Let us remove the fluid properties from the right side of Eq. 9 by noting that the tributary volume in a fluid of density $\rho_{f}$ is also a tributary mass $M_{t}$ in $\mathrm{kg} / \mathrm{m}^{2}$ as follows:

$$
M_{t}=h \rho_{f}
$$

Eq. 9 takes the form:

$$
\frac{\mu \rho_{f}}{P_{c}}=\frac{M_{t}}{d u / d r}
$$

Note how a tributary mass expansion rate is defined by the fluid properties. According to the conclusions of Eq. 2, we compute the tributary mass per unit velocity gradient equal to $1.148 \times 10^{-3} \mathrm{~kg}$ $\mathrm{s} / \mathrm{m}^{2}$. Let us denote the tributary mass per unit velocity gradient $\varphi$ as $\varphi=M_{t} /(d u / d r)$ and rewrite Eq. 11 as

$$
\frac{\mu \rho_{f}}{\varphi}=P_{f}
$$

As a result of simple conventional fluid mechanics we concluded that the shear stress will be opposed by a pressure gradient acting on a plane normal to the shear stress. The previous development appears to suggest that one can decide on the pressure gradient from the fluid properties and the dynamics by means of Eq. 12. The pressure gradient so derived is a unique value for the fluid properties, so that the computed shear stress equal to the viscosity when the velocity gradient is equal to unit and the velocity is satisfied simultaneously, hence, defining the size of the expansion about the shear stress.

In addition, working in further development, let us keep in mind the surface force nature of the pressure gradient and highlight that for unbounded water of density equal to $1,000 \mathrm{~kg} / \mathrm{m} 3$, the effect of gravity is the same as a pressure gradient of 9,807 $\mathrm{Pa} / \mathrm{m}$ so that the same consideration can be given to the body force $g$ to compute the force. Giving the same consideration to the conclusion of Eq. 12, for an unbounded fluid, we note that the pressure gradient mobilized by the viscous resistance (or deformation resistance) of $991 \mathrm{~Pa} / \mathrm{m}$ is less than the pressure gradient mobilized by gravity, hence, incapable of keeping a pressure head. In the same context, we note that the ratio $p_{c} / \rho_{f}$ is a body force $I_{f}$ in $N / \mathrm{kg}$ having the same effect as the pressure gradient, to rewrite Eq. 12 as:

$$
\frac{\mu}{I_{f}}=\varphi
$$

Although the term "body force" is being used, $I_{f}$ is also a pressure gradient. Instead of per meter, in the direction of the applied stress, is per kilogram distributed on the square meter, where the stress is being applied.

Equation 13 leads to a rational definition of the Newtonian limit, when $I_{f}=g$ as

$$
\mu=g \varphi
$$

For an unbounded Newtonian fluid, the build up of a pressure head is not possible until the viscosity overcomes $0.01126 \mathrm{~Pa}$-s. It is not expected that the relationships derived are applicable where the viscosity reaches this limit.

\section{VALIDATION}

In this section we note that Eq. 11 also defines the size of the expansion about a unit velocity gradient and hence the geometry. One can verify that the definition of the geometry is essential in the computation of the velocity based on the dynamics of viscosity. We hence conclude that the computation of the velocity based on the prescriptions defined by Eq. 11 is ultimately, impossible, unless, the developments on the previous section are based on a sound assessment of the dynamics. For the purpose of validation, one may try two approaches: one, using of measured settling velocities to find the pressure gradient and the tributary mass by means of Eq. 2, or two, use the principle right away to compute settling velocities (or the radius) and compare with experimental data. We consider the latter approach with data sets including identical particle sizes in different fluids and temperature.

The data sets include the following experimental values of settling velocities indicated on Table 2 :

For sand particles 0.5 to $25.0 \mu \mathrm{m}$ (Gs taken as 2.65) in water at $15 C^{o}$ from Zegzhda (1934), Arkangel'skii (1935) and Sarkisyan (1958) reproduced by Cheng, N. (1997).

The average settling velocity for sand particles 5.0 to $25.0 \mu \mathrm{m}$ ( $G$ s taken as 2.65) in water at $20 C^{\circ}$ from Raudkivi (1990), also reproduced by Cheng, N. (1997);

The hard sphere limit velocity at infinite dilution for identical organophilic silica hard spheres 0.031 to $0.037 \mu \mathrm{m}$ radius in cyclohexane and in toluene reported by Jansen et al. (1986)

The hard sphere limit velocities at infinite dilution reported by Davis et al. (1991) for hard spheres ranging from 0.09 to $0.21 \mu \mathrm{m}$ radius in cyclohexane at $25 C^{o}$.

The last two data sets are extrapolated values at infinite dilution from the concentration behavior of hard spheres in the colloidal range. 
Table 1. Fluid parameters. Viscosities taken from documents by Jansen et al. (1986) and Davis et al. (1991). The pressure gradient $P_{f}$ from Eq.11, the tributary volume $e_{\max }$ from Eq. 6 and the maximum spherical expansion $\xi_{\text {max }}$ as defined for Eq. 2.

\begin{tabular}{|c|c|c|c|c|c|c|c|}
\hline Fluid & $\mathrm{T}^{\circ} \mathrm{C}$ & $\begin{array}{c}\mu \\
(\mathrm{mPa}-\mathrm{s})\end{array}$ & $G_{f}$ & $\begin{array}{c}P_{f} \\
(\mathrm{~Pa} / \mathrm{m})\end{array}$ & $\begin{array}{c}G s \\
\text { Solids }\end{array}$ & $e_{\max }$ & $\xi_{\max }$ \\
\hline Cyclohexane & 25.0 & 0.898 & 0.774 & 605.4 & 1.8 & 16.62 & 5.31 \\
\hline Cyclohexane & 25.0 & 0.898 & 0.774 & 605.4 & 1.75 & 15.81 & 4.98 \\
\hline Toluene & 25.0 & 0.552 & 0.864 & 415.4 & 1.75 & 20.92 & 7.11 \\
\hline Water & 15 & 1.1390 & 0.9992 & 991.4 & 2.65 & 16.33 & 5.19 \\
\hline Water & 20 & 1.0030 & 0.9983 & 872.2 & 2.65 & 18.57 & 6.12 \\
\hline
\end{tabular}

Table 2. Comparison of reported radius by three techniques and computed from Eq. 2. Calculated radiuses from extrapolated hard sphere limit velocities Uo from Jansen et al. (1986) and Davis et al. (1991) and calculated radius from measured settling velocities from Zegzhda (1934), Arkangel'skii (1935) and Sarkisyan (1958) reproduced by Cheng, N. (1997); and from Raudkivi (1990), also reproduced by Cheng, N. (1997).

\begin{tabular}{|c|c|c|c|c|c|c|c|c|}
\hline No & Author & Fluid $/ \mathrm{T}^{\circ} \mathrm{C}$ & $\begin{array}{l}\text { Solids } \\
\text { Gs }\end{array}$ & $\begin{array}{l}\text { Uo } \\
(\mu \mathrm{m} / \mathrm{s})\end{array}$ & $\begin{array}{l}\text { TEM } \\
\text { rs } \\
(\mu \mathrm{m})\end{array}$ & $\begin{array}{l}\text { DLS } \\
\text { rs } \\
(\mu \mathrm{m})\end{array}$ & Measured & $\begin{array}{l}\text { Calculated } \\
\text { Eq. } 2 \\
\text { rs }(\mu \mathrm{m})\end{array}$ \\
\hline 1 & Raudkivi & Water / 20 & 2.65 & 1660 & & & 25 & 24.977 \\
\hline 2 & Arkangel'skii & Water / 15 & 2.65 & 1410 & & & 25 & 24.985 \\
\hline 3 & Raudkivi & Water / 20 & 2.65 & 414 & & & 12.5 & 12.473 \\
\hline 4 & Arkangel'skii & Water / 15 & 2.65 & 223 & & & 10 & 9.936 \\
\hline 5 & Raudkivi & Water / 20 & 2.65 & 66.3 & & & 5 & 4.992 \\
\hline 6 & \multirow[t]{3}{*}{ Arkangel'skii } & \multirow[t]{3}{*}{ Water / 15} & \multirow[t]{3}{*}{2.65} & 56.5 & & & 5 & 5.001 \\
\hline 7 & & & & 14.1 & & & 2.5 & 2.498 \\
\hline 8 & & & & 0.57 & & & 0.5 & 0.502 \\
\hline 9 & \multirow{6}{*}{$\begin{array}{l}\text { Davis } \\
\text { et al. }\end{array}$} & \multirow[t]{6}{*}{ Cyclohexane / 25} & \multirow[t]{6}{*}{1.80} & 0.1194 & 0.195 & & & 0.258 \\
\hline 10 & & & & 0.0750 & 0.14 & & & 0.205 \\
\hline 11 & & & & 0.0500 & 0.12 & 0.155 & & 0.167 \\
\hline 12 & & & & 0.0610 & 0.125 & 0.175 & & 0.185 \\
\hline 13 & & & & 0.0472 & 0.115 & & & 0.162 \\
\hline 14 & & & & 0.0250 & 0.08 & 0.1 & & 0.118 \\
\hline 15 & \multirow{2}{*}{$\begin{array}{l}\text { Jansen } \\
\text { et al. }\end{array}$} & Cyclohexane / 25 & \multirow[t]{2}{*}{1.75} & 0.00234 & 0.031 & 0.037 & & 0.037 \\
\hline 16 & & Toluene / 25 & & 0.00377 & 0.031 & 0.037 & & 0.038 \\
\hline
\end{tabular}

Here we choose to compute the radius from the given velocities. The choice is due to issues regarding the accuracy of the measuring techniques in the colloidal range as quoted by Wang et al. (2007), "Despite the fact that there exist several techniques capable of characterizing nanoparticle sizes, their measurement results from the same sample often deviate from each other by an amount that is considered significant on the nanometre scale", so that there is additional uncertainty on the actual particle sizes. Table 1 presents the fluid properties, the specific gravity of the solids, the computed pressure gradient and expansion and Table 2 presents the measured settling velocities for sand, the extrapolated hard sphere limit velocities Uo, the DLS (Dynamic Light Scatter) and the TEM
(Transmission Electron Microscopy) measurements; and the computed radiuses from Eq. 2 .

Note on the results on lines 1 to 8 the extreme agreement with the reported radiuses in water at 15 and $20 C^{o}$. Also to be highlighted, note the results on line 15 and 16 for identical organophilic silica particles in cyclohexane and toluene. Despite the substantial differences in expansion, viscosity and pressure gradient, Eq. 2 computes the same radius within a margin of $0.25 \%$.

In addition, Eq. 2 appears to overestimate the radius for the colloidal range, with very close agreement however, with the DLS measurements. The writer takes the opportunity to highlight a fact that make settling velocity measurements highly biased, especially, in the colloidal range (the experiments 
can last thousands of hours). The issue, to the knowledge of the author, has not been pointed out in the literature, neither the authors of the studied papers have shown concern about it. As a result of regularly conducting shallow refraction surveys, I'm very familiar with equipment induced (even very small equipment) ground vibrations and other sources of ambient noise, which include air noise ( $\mathrm{P}$ wave). In general, seismic surveys are conducted by placing receivers (geophones) on the ground. The time history of displacement of an oscillator, in the receiver, is displayed in a computer and can be recorded. Qualitatively, all buildings can be considered to be subject to a permanent time history of accelerations that can be considered to be very significant in the micron and submicron range. Fluids, at any location can in addition be subject to significant vibrations by air noise. In the context of our scale and dynamics, one should note that such a motion will induce an influential effect. On the grounds of our flow model, one can expect a greater settling rate due to the increase in the expansion that such motion would cause. The results in the colloidal range in Table 2 appear to suggest the expected influential effect of ambient vibrations, although, the outcome of the computation, regardless, is quiet notable.

In this section, we have finished the flow model for the settling velocity of spherical and non spherical particles in creeping motion derived in previous work. Further insights and improvement follows in the following section regarding non spherical particles.

\section{NON SPHERICAL PARTICLES}

In previous work, the following equation was derived for the settling velocity of non spherical particles:

$$
\begin{aligned}
& V_{s}=-\frac{p(r)}{\mu} \frac{2 b^{2} e_{\max }^{2}}{9(1+f(a, b))^{2}} \times \\
& \frac{\left(0.0001+\left(1+\frac{2 e_{1 \mathrm{max}}}{3}-\left(1+e_{1 \mathrm{max}}\right)^{\frac{2}{3}}\right)\right)}{\left(0.0001+\frac{e_{1 \max }^{2}}{9}\right)}
\end{aligned}
$$

Where $e_{1 \max }$ is the maximum tributary ratio $e_{\max }$ multiplied by the inverse of the aspect ratio of an oblate spheroid particle having dimensions $b$ for $1 / 2$ the thickness and $a$ for the radius. $F(a, b)$ is defined as $f(a, b)=(b / a) 3 / 2$. The factor 0.001 is just to remove the difficulty of the relationship turning into one when $e$ tend to $e_{\max }$ but undefined when $e$ is exactly $e_{\max }$ at the far end of the velocity profile. This difficulty only arises when computing the values for the entire profile. Simplification of Eq. 15 is hence simple when written as follows:

$$
\begin{aligned}
& V_{s}=-\frac{p(r)}{2 \mu} \frac{4 b^{2}}{9(1+f(a, b))^{2}} e_{\max }^{2} \times \\
& \frac{\left(1+\frac{2 b e_{\max }}{3 a}-\left(1+\frac{b e_{\max }}{a}\right)^{\frac{2}{3}}\right)}{\frac{b e_{\max }^{2}}{9 a}}
\end{aligned}
$$

As written in Eq. 15 is of greater interest when considering its derivation. From its form, in Eq. 16, we note the first ratio, which has a factor of $1 / 2$, as a consequence of the integration, is the same ratio that appears in Eq.2. The second ratio is the volume to area ratio squared of the particle and the third ratio is the ratio of the maximum spherical expansion to the maximum non spherical expansion. Eq. 16 can be verified to turn into Eq. 2 when the particle is a sphere. It can also be further simplified. The computation in Eq. 16 also relies in an accurate characterization of the driving force, via specific surface area $S S A$ and geometry that correlates accurately to the $S S A$.

Equation 15 was derived as a result of the insights of the impact of the shape and magnitude of the driving force on the geometry and the size of the expansion. In this approach, we derived a relationship to compute the shear stress across a tributary volume that is neither planar, nor spherical and conducted the integration to compute the velocity.

Using the same understanding the following alternative approach is proposed to derive a relationship to compute accurate settling velocities for non spherical particles. Consider an oblate spheroid of dimensions $a$ and $b$ of known specific gravity having a volume $V_{o}=4 / 3 \pi a^{2} b$ and surface area $A_{o}=2 \pi a^{2}\left(1+(b / a)^{3 / 2}\right)$ say in water at $20 C^{o}$. The volume of fluid retained in the ambient fluid $V_{f}$ can be computed as $V_{f}=e_{\max } V_{o}$ and a pseudo sphere of radius $r_{s p}$ having the same surface area and wall shear of the non spherical particle can be found as $\left.r_{s p}=a\left(\left(1+(b / a)^{3 / 2}\right)\right) / 2\right)^{1 / 2}$. From our definitions the maximum pseudo tributary volume $e_{\text {pmax }}$ follows as

$$
\begin{gathered}
e_{p \max }=\frac{\frac{4}{3} \pi a^{2} b e_{\max }}{\frac{4}{3} \pi a^{3}\left(\frac{\left(1+\left(\frac{b}{a}\right)^{3 / 2}\right)}{2}\right)^{3 / 2}} \Rightarrow \\
e_{p \max }=\frac{2^{3 / 2} b e_{\max }}{a\left(1+\left(\frac{b}{a}\right)^{3 / 2}\right)^{3 / 2}} \Rightarrow
\end{gathered}
$$


One can hence compute the settling velocity Vsp of the pseudo sphere of radius $r_{s p}$ that settles at the same velocity Vnsp of the non spherical particle as

$$
\begin{aligned}
& V_{n s p}=\frac{-P_{f} r_{s p}^{2}}{2 \mu} \times \\
& \left(1+\frac{2 e_{p \text { max }}}{3}-\left(1+e_{p \text { max }}\right)^{\frac{2}{3}}\right)
\end{aligned}
$$

The pseudo expansion so obtained is just a mean to model the flatter expansion mobilizing the same shear stress as the non spherical particle. In the latter approach, we note that the pseudo sphere is quiet large but this can be done with impunity as Eq. 18 is a computation within the geometry of the ambient fluid, as it relates to the driving force, not the solid sphere. The computation using the pseudo sphere is in very close agreement with Eq. 16 and the corresponding experimental data but it offers a simpler and improved rational to model the tributary volume.

Based on the fact that there is always a sphere of radius $r_{s}$ that settles at the same velocity as the non spherical particle, a chart was developed for the thickness and aspect ratio of all particles settling at a given velocity shown in Figure 1.

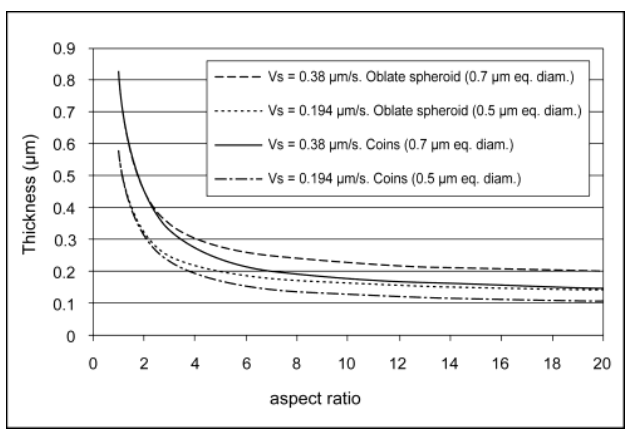

Fig. 1. Combination of aspect ratio thickness of particles with Gs $=2.62$ settling at 0.380 and $0.194 \mu \mathrm{m} / \mathrm{s}$ for coins and oblate spheroids.

Using the same approach we note

$r_{p}^{2} \xi_{p \max }=r_{s}^{2} \xi_{\max }$

This can be used to construct the same chart using the definitions explained above.

Equation 2, via expansion, pseudo expansion and pseudo radius can be used to solve for the settling velocity for a wide variety of geometries. Other than oblate spheroids and cylinders, previously discussed, polyhedra, cylinders, oblate spheroids and other geometries can be considered by means of Eq. 16 provided accurate characterization of the geometry, specific surface area and how they influence the expansion are used. It is also overstressed that although, the volume to area ratio of the particle is of great significance, as in fact correlates exactly with the radius for spheres, the volume to area ratio should not be used, as is, on Eq.2 (Using the expansion or the pseudo expansion), for non spherical particles as such substitution implies a complete break down of the relationships controlling the dynamics of the ambient fluid about settling objects.

As a corollary, note that the rational provided in this flow model provides the grounds to develop the relationships for a solution from the cylinder that extends to infinite and a stream function for both, the sphere and the infinite cylinder. The readers are encouraged to undertake the task.

\section{CONCLUSION}

A flow model for the settling velocity of spherical and non spherical particles have been presented. The model, allows a rational approach to embrace a wide range of sedimentation problems with a wide perspective. The model is expected to contribute to further developments for isolated particles, hindered sedimentation, permeability, the sedimentation of aggregates, etc.; where further developments are needed.

\section{ACKNOWLEDGMENTS}

To my father Luis Paulino Mendez 1925-2012 who provided unconditional support to his children and family with lifetime sacrifices. To JAFM for providing comprehensive feedback for enhancement of previous work. This paper has been written entirely on the budget and time of the author.

\section{REFERENCES}

Al-Naafa, M. A., M. S. Selim (1992). Sedimentation of monodisperse and bidisperse hard-sphere colloidal suspensions. AIChE Journal, American Institute of Chemical Engineers 38(10), 1618-1630.

Arkangel'skii, B. V., 1935, Experimental Study of Accuracy of Hydraulic Coarseness scale of Particles. Isv. NHG 15, Moscow, Russia (in Russian).

Cheng, N. (1997). Simplified Settling Velocity Formula for Sediment Particle. Journal of Hydraulic Engineering 123(2), 149-152.

Davis, K. E., Russel, W. B. and Glantschnig, W. J., (1991), Settling suspensions of colloidal silica: observations and X-ray measurements. Journal of the Chemical Society, Faraday Transactions. Vol. 87, pp. 411-424.

Happel J. and Brenner, H. (1983). Low Reynolds Number Hydrodynamics, third Ed. Martinus Nijhoff Publishers, The Hagge, The Netherlands.

Jansen, J. W., De Kruif, C. G., Vrij, A., (1986), Attractions in Sterically Stabilized Silica Dispersions, Journal of Colloid and Interface Science. Vol. 114, No. 2, pp. 501-504.

Lu, N., G. Ristow and W. Likos (2000). The accuracy of hydrometer analysis for fine-grained clay particles. Geotechnical Testing Journal 33(4), 487-495. 
Mendez, Y. (2011). A flow Model for the Settling Velocity of non Spherical Particles in Creeping Motion. Journal of Applied Fluid Mechanics, Vol. 4, No. 4, pp. 65-75, 2011.

Pruett, R. and H. Webb (1993). Sampling and analysis of KGa-1 B well-crystallized kaolin source clay. Clays and Clay Minerals. 41(4), 514-519.

Raudkivi, A. J. (1990). Loose Boundary Hydraulics, $3^{\text {rd }}$. Ed. Pergamon Press Inc., Tarritown, N. Y.

Sarkisyan, A. A. (1958). Deposition of sediment in a turbulent stream. Izd. AN SSSR, Moscow, Russia (in Russian).

White, F. M. (1994). Fluid Mechanics $3^{\text {rd }}$ ed., New York, McGraw-Hill, Inc.

Winter, H. (2011). National Science Foundation: Division of Chemical, Bioengineering, Environmental, and Transport Systems. Fluid Dynamics. Program guidelines to PD 12-1443. September 15, 2011. <http://www.nsf.gov/funding/pgm_summ.jsp?pi ms_id=13365>

Zegznda, A. P. (1934). Settlement of sand gravel particles in still water. fev. NIIG 12, Moscow, Russia (in Russian).

Zhu, L.J., and N. S. Cheng (1993). Settlement of sediment particles. Research Report, Department of River and Harbor Engineering, Nanjing Hydraulic Research Institute, Nanjing, China (in Chinese). 